\title{
The relationship between age, gender and the dark triad of personality
}

\author{
Foram Makim ${ }^{1}$, Vidyadayini Shetty ${ }^{2}$ \\ ${ }^{1}$ TYBA student, ${ }^{2}$ Associate Professor \\ Department of Psychology, Nagindas Khandwala College, Mumbai. \\ Corresponding author: Foram Makim \\ Email - forammakim@gmail.com
}

\begin{abstract}
Background: Dark triad refers to a set of traits that include the tendency to seek admiration and special treatment (otherwise known as Narcissism), to be callous and insensitive (Psychopathy) and to manipulate others (Machiavellianism). The purpose of this study was to study the difference in dark triad amongst individuals with different age groups and gender.

Methods: Convenient sampling was used to collect the data. A total of 90 participants (44 males and 46 females) participated in the study with consent. The respondents belonged to three age groups: early adulthood (20-34), midlife (35-49) and mature adulthood (50-64). A 27-item version of The Short Dark Triad (SD3) was used for the assessment of dark triad.

Results: Descriptive statistics, t-test and one way ANOVA was used to analyze the data. The findings indicate that there is no significant difference in Narcissism and Machiavellianism between males and females however there is a significant difference in Psychopathy between males and females. Also, the F value indicate that there is a significant difference in the three personality traits in reference to different age groups. Post hoc analysis indicates a significant difference in psychopathic behavior between 20-35yrs (early adulthood) and 36-49 (mid life adulthood) age groups and between 20-35yrs and 50-64yrs (mature adulthood) age groups. However there is no significant difference in psychopathic behavior between 3649yrs and 50-64yrs age group.

Conclusion: Males score higher on psychological tendencies as compared to females. Secondly, psychopathic tendencies are more in participants who are in early adulthood (20-34) than people in midlife (35-49) and mature adulthood (50-64).
\end{abstract}

Keywords: Narcissism, Machiavellianism, Psychopathic Behavior, Dark triad, Age, Gender.

(Paper received $-30^{\text {th }}$ December 2017 , Peer review completed $-4^{\text {th }}$ January 2018)

(Accepted $-6^{\text {th }}$ January 2018)

\section{INTRODUCTION}

Dark triad refers to a set of traits that include Narcissism, Machiavellianism and Psychopathy. The components of the Dark Triad are considered maladaptive personality traits that include Narcissism, Psychopathy, and Machiavellianism. Narcissism is characterized by an exaggerated sense of entitlement, grandiosity, and superiority. The second component of the triad is Psychopathy, described by low anxiety and empathy and high thrill seeking behaviour. Finally, the last component is Machiavellianism, consisting of highly manipulative individuals characterized by interpersonal coldness Research on the Dark Triad initially began by observing clinical and subclinical samples on various personality traits and it was found that Narcissism, Psychopathy, and Machiavellianism were the most frequent maladaptive personality traits [1-2]. There is a small body of research which highlights how the so-called dark traits can 
help one to be successful in life. The authors hence decided to investigate the degree of the three dark traits: Narcissism, Machiavellianism and Psychopathy in males and females of different age groups [2].

\section{Hypothesis}

There is no difference in the degree of Narcissism, Machiavellianism and Psychopathy between males and females.

There is no difference in the degree of Narcissism, Machiavellianism and Psychopathy amongst different age groups.

\section{Sample}

\section{METHODOLOGY}

The sample consisted of total 90 participants (44 males, 46 females). The sample was divided into 3 different age groups: early adulthood (20-34), midlife (35-49) and mature adulthood (50-64).

\section{Tools used}

The 27-item version of The Short Dark Triad (SD3) developed by Jones and Paulhus, 2013 [1] was used for the assessment of dark traits. All items were presented in Likert-type format with anchors 1 (strongly disagree) to 5 (strongly agree). After recoding the Reversal (indicated with "R") and adding the total scores, each subscale was formed by adding the items. The SD3 reliabilities were; Mach $\alpha=0.74$, Psychopathy $\alpha=0.72$, narcissism $\alpha=0.68$. The SD3 validities were $0.82,0.92$, and 0.87 for Narcissism, Machiavellianism, and Psychopathy respectively.

\section{Procedure}

Convenient sampling method was used to collect the data. Interested respondents were approached. Without disclosing the aim of the study, participants between 20-64years who were willing to participate were asked to fill the questionnaire. The sample consisted of younger and older generation who were single, married or had grandchildren.

\section{RESULTS}

Descriptive statistics, $t$ test and one way ANOVA was used to analyze the data collected. The scores obtained from the questionnaires were manually assessed and crosschecked using Microsoft Excel to avoid human errors. All the 90 items in the questionnaire were grouped into their relevant age groups and gender and the summation of the scores was done accordingly.

Table 1 - Means, SDs, and $t$ values of the dark triad scores of males and females

\begin{tabular}{|l|l|l|l|l|l|l|l|}
\hline & \multicolumn{3}{|l|}{ Narcissism } & \multicolumn{2}{l|}{ Machiavellianism } & \multicolumn{2}{l|}{ Psychopathy } \\
\hline & N & Mean & SD & Mean & SD & Mean & SD \\
\hline Males & 44 & 27.48 & 3.80 & 28.0 & 3.51 & 19.80 & 4.34 \\
\hline Females & 46 & 26.35 & 4.71 & 27.43 & 5.67 & 21.80 & 4.98 \\
\hline t-value & & 1.25 & 0.57 & -2.04 & \\
\hline p-value & & 0.2149 & 0.5732 & -0.0447 & \\
\hline & NS & NS & Sig & \\
\hline
\end{tabular}

Table 1 reflects mean scores on Narcissism of males and females which was found to be 27.48 and 26.35 and the corresponding SD was 3.80 and 4.71 respectively. The $t$-value $(t=1.25, p>0.5)$ indicate no significant difference between mean scores of males and females on Narcissism. The mean scores on Machiavellianism of males and females was found to be 28.0 and 27.43 and the corresponding SD was found to be 3.51 and 5.67 respectively. The $t$-value $(t=0.57, p>0.5)$ indicate no significant difference between mean scores of males and females on Machiavellianism. The Mean scores on Psychopathic behavior of males and females was found to be 19.80 and 21.80 and the corresponding SD was found to be 4.34 and 4.98 respectively. The $\mathrm{t}$-value $(\mathrm{t}=-2.04, \mathrm{p}<0.5)$ was found to be statistically significant. In other 
words, there was a significant difference between the mean scores of males and females on Psychopathic behavior.

Table 2 - Means, SDs, F values of the dark triad scores of the three age groups

\begin{tabular}{|c|c|c|c|c|c|c|c|c|c|c|c|}
\hline & \multicolumn{3}{|c|}{$20-34 y r s$} & \multicolumn{3}{|c|}{$35-49 \mathrm{yrs}$} & \multicolumn{3}{|c|}{$50-64 \mathrm{yrs}$} & \multirow{2}{*}{$\begin{array}{l}\text { F- } \\
\text { value }\end{array}$} & \multirow[t]{2}{*}{ p-value } \\
\hline & $\mathrm{N}$ & Mean & SD & $\mathrm{N}$ & Mean & SD & $\mathrm{N}$ & Mean & SD & & \\
\hline Narc & 31 & 27.1 & 4.26 & 30 & 26.2 & 4.94 & 29 & 27.3 & 3.65 & 0.55 & 0.5767 \\
\hline Mach & 31 & 28.5 & 5.16 & 30 & 27.2 & 4.34 & 29 & 27.5 & 4.67 & 0.61 & 0.5461 \\
\hline Psych & 31 & 22.6 & 4.42 & 30 & 19.7 & 5.00 & 29 & 20.1 & 4.42 & 3.74 & 0.0278 \\
\hline
\end{tabular}

The F values for Narcissism and Machiavellianism indicate no significant difference among the three age groups. However, the F value for Psychopathic behaviour indicates significant difference for the three age groups. Hence a Post Hoc test was used. The post hoc analysis indicates a significant difference in the scores of Psychopathic behaviour between people in the early adulthood and people in mid-life. Similarly, people in early adulthood have significantly higher scores in psychopathic behaviour as compared to mature adults.

\section{DISCUSSION}

The key finding in this study was that males have more tendencies to adopt psychopathic behavior than females as compared to Narcissism and Machiavellianism. Using a Meta-Analytic method Muris et al., 2017, identified 50 studies that included a total of 65 samples containing 25,930 participants in which gender differences for the three dark triad traits were investigated. The findings indicate that for all three traits positive and statistically significant effect sizes were found, confirming the idea that men in general display higher levels of Narcissism, Machiavellianism, and Psychopathy relative to women. However, the present study did not obtain a significant difference in the dark triad among males and females in Narcissism and Machiavellianism [2-3].

The other finding was that people in their early adulthood (20-34 years) tend to have more psychopathic traits than people in midlife (35-49 years) and mature adulthood (50-64 years). This is because people in their early adulthood are reckless and immature than the latter group of people. Past studies that Narcissism and Machiavellianism prove to be useful for working adults between 25-34yrs as Machiavellianism was positively correlated to leadership positions and job satisfaction, and narcissism was strongly related to higher salaries. People high in these two traits were found to be more ambitious and intelligent. Although Psychopaths can be charismatic, intelligent and creative, they tend not be good team players, which seems to inhibit them from advancing quickly through the workplace. It does imply that they may work very well on their own, however. A recent review by Lilienfeld, Watts and Smith on "Successful psychopathy" notes that there are many psychopaths in leadership positions, so clearly, they can advance through the workplace on occasion. It suggests that the more successful psychopaths are more moderate and conscientious than others. In addition to this, successful psychopaths may be able to use their ability to not feel remorse or empathy as protective measures against other antisocial people [4].

\section{CONCLUSION}

The present studies indicate males score higher on psychological tendencies as compared to females. Further the findings point out that psychopathic tendencies are prevalent in participants who are in early adulthood (20-34) than people in midlife (35-49) and mature adulthood (50-64).

\section{REFERENCES}

1. Jones D, Paulhus D. (2013). The 27-item version of The Short Dark Triad scale (SD3) [Internet]. Available from: https://illimitablemen.com/2013/11/17/understanding-the-dark-triad/ 
2. Muris P, Merckelbach H, Otgaar H, Meijer E. The malevolent side of human nature: A meta-analysis and critical review of the literature on the Dark Triad (Narcissism, Machiavellianism, and Psychopathy). Persp Psychological Sci 2017;12(2):183-204.

3. Stenason L. Implicit and Explicit Self-Esteem in Relation to the Dark Triad. Western Undergraduate Psychology Journal 2014;2(1):12.

4. Lilienfeld SO, Watts AL, Smith SF. Successful psychopathy: A scientific status report. Curr Dir Psychol Sci 2015;24(4):298-303.

$* * * * * * * * * * * * * * * * * * * * * *$

Acknowledgements - Nil

Conflict of Interest - Nil

Funding - Nil 